\title{
INCUBADORAS SOCIAIS EN LAS UNIVERSIDADES COMO PROMOTORAS DE INNOVACIÓN Y EMPREENDIMIENTO SOCIAL PARA EL DESARROLLO DE LAS REGIONES: EL CASO DEL ESTADO DE HIDALGO, MÉXICO
}

\section{SOCIAL INCUBATORS IN UNIVERSITIES AS DRIVERS OF INNOVATION AND SOCIAL ENTREPRENEURSHIP FOR REGIONAL DEVELOPMENT: THE CASE OF THE STATE OF HIDALGO, MEXICO}

\author{
Elba Mariana Pedraza Amador ${ }^{1}$ \\ Maribel González Cadena ${ }^{2}$ \\ Judith Alejandra Velázquez Castro ${ }^{3}$
}

\section{RESUMO}

As universidades, a partir do ponto de vista social, têm a missão de acompanhar e contribuir, por meio da criação de conhecimento e formação de profissionais, na transformação rumo a uma sociedade equitativa e justa. O presente trabalho descreve o papel das universidades através das incubadoras sociais, para promover a inovação e o empreendimento social em zonas marginadas no México, a fim de facilitar o desenvolvimento sustentável e a reativação da economia melhorando o bem-estar da sociedade. Nesse sentido, no estado de Hidalgo, em diversas universidades operam programas de apoio ao setor social e produtivo através destas incubadoras. A partir de uma revisão documental se identificam as principais ações desenvolvidas pelas mais importantes incubadoras sociais do estado de Hidalgo durante os três últimos anos de operação. O resultado permite reconhecer o trabalho realizado pelas incubadoras sociais das universidades em atenção às problemáticas sociais da entidade, tais como inclusão de pessoas com deficiência e o empreendimento em regiões indígenas promovendo o planejamento e implementação de melhores maneiras de cobrir necessidades de coletivos empobrecidos e marginados, o que permite favorecer o crescimento pessoal e coletivo das regiões e da equidade.

Palavras-chave: Incubadoras sociais. Empreendedorismo. Inovação social.

\begin{abstract}
Universities - from the social point of view - have the mission to monitor and contribute in the transformation towards an equal and fair society through the creation of knowledge and training of professionals. This paper describes the role of universities through social incubators to promote innovation and social development in remote areas in Mexico, in order to facilitate sustainable development and the reactivation of the economy by improving the well-being of society. In this sense, in the state of Hidalgo, several universities operate programs to support social and productive sectors through these incubators. From a documentation review, we identified the main actions developed by the most important social incubators of the State of Hidalgo during the last three years of operation. The results allow us to recognize the work done

\footnotetext{
${ }^{1}$ Universidad Autónoma del Estado de Hidalgo, México. Edificio Torres de Rectoría, Carr. Pachuca, Actopan, Km. 4.5, $7^{\circ}$ piso, Colonia Campo de Tiro, 42039, Pachuca, México. E-mail: elbam@uaeh.edu.mx

${ }^{2}$ Universidad Autónoma del Estado de Hidalgo, México. Edificio Torres de Rectoría, Carr. Pachuca, Actopan, Km. 4.5, $7^{\circ}$ piso, Colonia Campo de Tiro, 42039, Pachuca, México. E-mail: maribelglez@ hotmail.com

${ }^{3}$ Universidad Autónoma del Estado de Hidalgo, México. Edificio Torres de Rectoría, Carr. Pachuca, Actopan, Km. 4.5, $7^{\circ}$ piso, Colonia Campo de Tiro, 42039, Pachuca, México. E-mail: judith_veca@hotmail.com
} 
by social incubators in universities regarding social issues of the entity, such as inclusion of people with disabilities and entrepreneurship in indigenous regions, promoting planning and implementation of the best ways to cover the needs of impoverished and remote communities, allowing the fostering of personal and community growth of regions and equity.

Keywords: Social incubators. Entrepreneurship. Social innovation.

\section{Introducción}

En los últimos años las universidades se sitúan en una posición social estratégica por su participación en la economía del conocimiento, ya que realizan de forma creciente lo que se ha denominado la tercera misión de la universidad (BUENO, 2007), la cual se orienta a la necesidad de saber aplicar la ciencia y responder a la demanda social de su tiempo, es decir un compromiso de la universidad como agente de creación y transferencia de conocimiento. La tercera misión de la Universidad representa una nueva forma de entender la investigación y la innovación pero sobretodo, promueve la transmisión fuera del ámbito universitario, para lo que precisa de una estrecha colaboración entre los distintos agentes del sistema (gobierno, empresas, otras Instituciones de Educación Superior y la sociedad) (GALINDO, 2010), debido a que tiene como objetivos: la responsabilidad social institucional de la universidad y el compromiso de valorizar la investigación y fomentar proyectos emprendedores o llevar a cabo proyectos de desarrollo territorial en colaboración con el resto de los agentes del sistema económico (VILALTA, 2000).

En el momento actual la universidad ha de ser motor de la competitividad regional al fungir como promotora del valor intelectual, tecnológico y científico y que permita redireccionar la innovación hacia las necesidades de la sociedad actual.

Por otra parte, el emprendimiento empresarial y social se ha convertido en un foco de atención al ser un medio para la generación de empleo, ingresos e innovación, que requiere la participación y convergencia entre universidad, gobierno, empresa y sociedad; ya que al involucrar al sector social en los procesos de emprendimiento se fortalecen los niveles de competitividad empresarial, contribuyendo así al desarrollo de una región o país al buscar el bienestar de la comunidad involucrada lo que coadyuva a la reducción de la exclusión social (SALINAS, 2012). De hecho, el emprendimiento social se enfoca en la innovación y el cambio social; debido a que son empresas que necesitan fondos para cumplir su objetivo social y el surgimiento del mismo obedece a factores como las modificaciones en las políticas de bienestar social, crecientes 
incentivos a la innovación social, creciente demanda de servicios sociales y comunitarios y el auge de organizaciones nacionales e internacionales con objetivos sociales (MARTÍNEZ; RODRÍGUEZ, 2013).

De acuerdo con el Índice de Condiciones Sistémicas para el Emprendimiento Dinámico, México es el segundo país de Latinoamérica para emprender, sólo por debajo de Chile, sus principales fortalezas son las políticas y regulaciones, las condiciones de la demanda y el capital social con el que cuenta, sin embargo, sus principales debilidades son la cultura emprendedora, el capital humano emprendedor y la plataforma de ciencia y tecnología para la innovación (KANTIS, 2014). Por ello, en México, el gobierno con el objetivo de participar como facilitador, inversor e integrador en el ecosistema de innovación crea el Instituto Nacional del Emprendedor que busca impulsar la innovación, competitividad y proyección en los mercados nacional e internacional de las micro, pequeñas y medianas empresas para aumentar su contribución al desarrollo económico y bienestar social (INADEM, 2014). Ya que según datos oficiales en el país, una empresa que abre de manera independiente tiene el $27 \%$ de posibilidades de tener éxito después de dos años de operación (CONSEJO DE COMUNICACIÓN, 2014).

En este sentido, las incubadoras de empresas tienen como objetivo el impulso, creación y desarrollo de las empresas en sus primeras etapas de vida, brindando apoyo a los nuevos empresarios (GARCÍA, 2012).

En México pertenecen al ecosistema emprendedor 204 instituciones de educación superior públicas y 124 instituciones privadas las cuales cuentan con algún programa de apoyo al emprendimiento, principalmente a través de modelos de incubación con la finalidad de guiar a los emprendedores en el proceso de operación y para ello requieren de contar con un plan de negocios, prototipos de los productos y servicios y que la empresa esté constituida, definir la inversión que necesitan para iniciar, los planes de trabajo así como analizar si requieren buscar capital, fases que son primordiales en las primeras fases de vida de la empresa y que tienen su apoyo en los procesos de incubación.

En el estado de Hidalgo, 56 municipios de 84 presentan un índice de marginación muy alto a medio (COESPO, 2014), motivo por el cual en los últimos años se han promovido apoyos a emprendedores sociales privilegiando los relacionados con el campo, la inclusión y otros proyectos productivos con el fin de mejorar el bienestar 
de la población de allí que se tome como caso de estudio del presente documento para analizar la labor que realizan las principales incubadoras sociales del estado fundamentalmente aquellas ligadas a universidades.

\section{Emprendimiento social e innovación}

El modelo del sistema económico global actual se caracteriza por estar asentado en el paradigma de la competencia donde se privilegia el uso de las nuevas tecnologías y que lejos de resolver muchos de los problemas que ya existían en la economía mundial los ha agudizado tales como la desigualdad, pobreza, problemas medioambientales, de exclusión entre otros.

Por emprendimiento se entiende "la creación y consolidación de empresas a partir de la detección y explotación de oportunidades en los mercados (SHANE; VENKATARAMAN, 2000), de acuerdo con Salinas (2012) se refiere al conjunto de actitudes y conductas que dan lugar a un determinado perfil personal orientado hacia la autoconfianza, creatividad, capacidad de innovación, sentido de responsabilidad y manejo de riesgo por lo que la acción emprendedora está orientada al logro de objetivos mediante la puesta en marcha de un sistema organizado de relaciones interpersonales y combinación de recursos.

La acción emprendedora se puede desarrollar en diversos ámbitos: a) empresarial cuando la principal motivación de sus acciones e iniciativas son de carácter económico; b) social cuando se propone dar respuesta a necesidades sociales al promover los conocimientos, capacidades y recursos al servicio de la sociedad y c) público cuando la iniciativa es con motivación fundamentalmente política o se intenta trasladar la cultura de la empresa al sector público (MORIANO, 2005).

Por lo anterior aunque el término de emprendimiento social siga en debate a pesar de su auge, se observa que un elemento común es la búsqueda de soluciones a problemas sociales, ya que el emprendedor social identifica oportunidades que se presentan a sí mismas como problemas que requieren soluciones y se esfuerza por crear emprendimientos (SULLIVAN, 2007). Diversos autores (ROBERTS; WOODS, 2005; MARTIN; OSBERG, 2007) hacen una aclaración con respecto a que la actividad de emprendimiento social no es necesariamente sin ánimo de lucro sino que está profundamente arraigada en dar a otros, es decir en crear un valor social sostenible 
fuertemente influenciado por el deseo de cambio social y de sostenibilidad de la organización y los servicios sociales que presta (GUZMÁN; TRUJILLO, 2008).

Para su identificación los emprendimientos sociales tendrían una motivación en común tal como la conciencia de un problema social y la voluntad de cambiarlo por lo que las empresas, con diferente tamaño, forma jurídica y ámbito de actuación han utilizado el mercado para alcanzar un objetivo de cambio social. Y es que la creación de valor social puede entenderse como el fin último y la razón de ser del emprendimiento social pero usando para ello herramientas propias de las empresas convencionales que son guiadas por la creación de valor económico como objetivo primario.

Sin embargo se pueden tomar como referencia algunos criterios para la identificación de emprendimientos sociales (MERINO, 2013) tales como:

- Definición de una misión para crear y mantener valor social.

- Compromiso de participación en un proceso de innovación aprendizaje continuo y adaptación al entorno.

- Reconocimiento de nuevas oportunidades.

Los actores que pueden intervenir en actividades o iniciativas se emprendimiento social son diversos tal como se ve en la figura 1.

Figura 1 - Agentes en el emprendimiento social

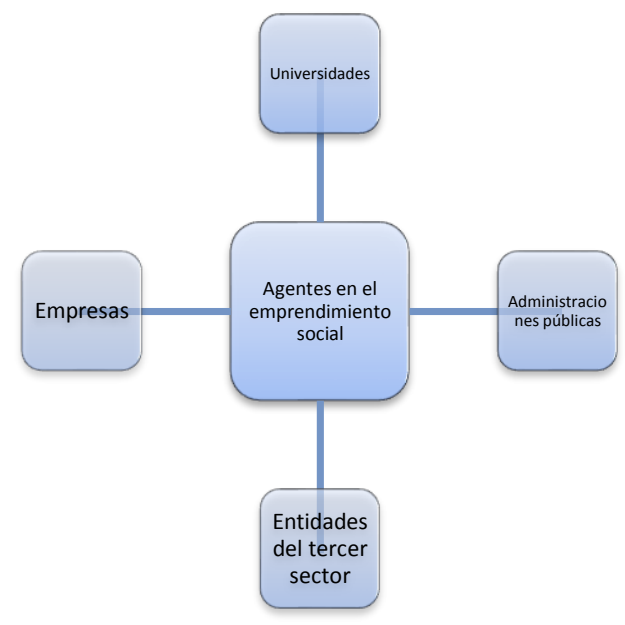

Fuente: elaboración propia (2014).

En cada caso el emprendimiento social incluye a los emprendedores que van más allá de una mera gestión empresarial responsable ya que no sólo inducen el cambio 
sino que actúan de modo directo para la transformación de la sociedad y/o promover y facilitar el cambio social en otros agentes, que puedan coparticipar en la producción, distribución y consumo y fomentar el empoderamiento de la sociedad civil y la colaboración solidaria como forma predominante de las relaciones sociales. Lo anterior da lugar a que las organizaciones creadas y los problemas que se pretenden abordar sean muy variados entre los que destacan: la sanidad, el acceso al agua y su salubridad o el apoyo a actividades agrícolas de zonas rurales, apoyo a la cultura, proveer servicios a las personas discapacitadas, colaborar en procesos de recogida de residuos y protección del medioambiente, entre otras.

De acuerdo con Curto (2012) se pueden identificar cuatro tipos de empresas sociales dependiendo del enfoque si está centrado en el lucro o no (ver Figura 2).

Figura 2 - Tipología de las empresas sociales
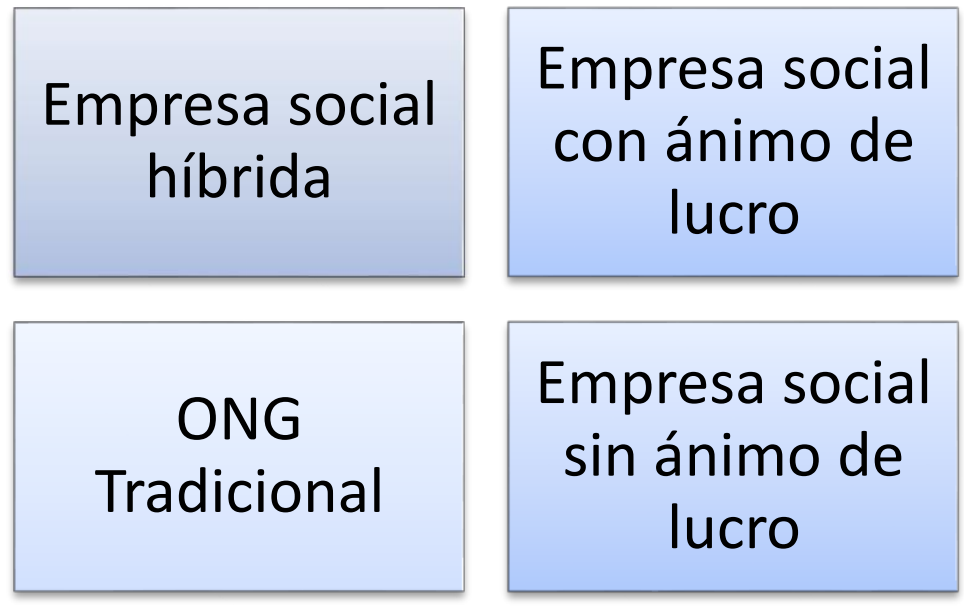

Fuente: elaboración propia (2014).

Según Schwab pueden clasificarse como:

a) Empresas apalancadas sin ánimo de lucro, cuya principal fuente de financiamiento es a través de las donaciones filantrópicas.

b) Empresas sin ánimo de lucro híbridas, cuyo modelo incluye cierta recuperación de costes a través de la venta de bienes y servicios y se suelen fundar entidades legales para depositar los ingresos y hacer uso de los gastos de una forma más eficiente. 
c) Organizaciones empresariales sociales, en cuyo caso la organización es con ánimo de lucro para proveer servicios o productos sociales o ecológicos. Para su financiamiento suelen buscar inversores.

Por otra parte los emprendimientos sociales además de diseñar un proyecto $\mathrm{u}$ organización que genere mejoras sociales sobre un determinado sector de la sociedad, también debe de hacerlo llegar al mayor número de personas posible de tal forma que las innovaciones sociales se difundan de forma óptima para ello requieren de una estructura capaz de movilizar recursos y personas, elaborar un programa con acciones que persigan un fin específico y definir las pautas, valores y principios que orienten el desarrollo del proyecto social así como identificar las vías para la captación de recursos financieros.

Por ello los emprendedores sociales tienen un perfil y características que los distingue tales como una visión emprendedora para realizar sus proyectos, una idea innovadora que busca producir un cambio social específico aplicando acercamientos prácticos, innovadores y sostenibles que beneficien a la sociedad con énfasis en sectores sociales marginados (ENCISO, 2012). De allí que la innovación aplicable, al ámbito social, sea otro factor común a estos emprendimientos.

\subsection{Innovación social}

La innovación es un proceso que, aunque ha sido tradicionalmente asociado a la creación o mejora de productos y servicios, es aplicable a otros aspectos como la reinvención de los procesos de negocio, la creación de nuevos mercados o los cambios en el uso de los canales de distribución, entre otros. Sin embargo, la innovación social es considerada como un "proceso complejo de introducción de nuevos productos, procesos o programas los cuales cambian las rutinas básicas, los flujos de recursos y autoridad, o bien las creencias del sistema social en el cual esta innovación ocurre" (WESTLEY; ANTANDZE, 2010) es decir que pretende diseñar e implementar mejores maneras de cubrir necesidades sociales, sea de una forma completamente nueva o realizando combinaciones de elementos existentes para obtener resultados nuevos, es decir de generar el máximo valor para la sociedad con la menor cantidad de recursos para cubrir las carencias de la sociedad en cada momento. 
De acuerdo con el Centre de recherche sur las innovations sociales (OCDE, 2010) la innovación social es una intervención iniciada por actores sociales que responden a una inspiración de proveer por una necesidad, el beneficio desde la oportunidad de modificar relaciones sociales al transformar patrones establecidos de comportamiento o proponen nuevas orientaciones culturales. Por lo que se puede apreciar que trasciende sectores, niveles de análisis e incluso los métodos para descubrir los procesos que produzcan un impacto duradero.

Lo anterior ligado al hecho de que uno de los motores de la innovación social tiene su origen en el hecho de que existen necesidades sociales y medioambientales no cubiertas, problemas que se intensifican desde la diversidad al conflicto, cambio climático; modelos que están fallando o se están estancando y áreas con nuevas posibilidades que no están explotando adecuadamente tales como el uso de las tecnologías. Por lo que uno de los principales factores que requiere la innovación social es conocer su misión o ser una organización basada en una misión; ya que la misión es un factor diferenciador clave para las empresas sociales pues es el que impulsará el éxito y permite el desarrollo de una cultura de innovación que sólo podrá ser palpable cuando ésta tenga lugar de forma coherente con los elementos constitutivos y la misión de la organización, respondiendo a un objetivo social que funciona de forma efectiva creando valor social y dirigiéndose de forma inherente a una necesidad social (RODRÍGUEZ et al., 2012).

La innovación social, presenta una serie de características específicas que deben ser tomadas en cuenta, de acuerdo con Bueno (2011):

- $\quad$ Que existe un agente que difunde y pone en común la innovación.

- Que requiere de un método interdisciplinar para encontrar la solución a las problemáticas sociales.

- Que la innovación social tiene un carácter permanente

- $\quad$ El resultado del proceso de innovación social debe ser la generación de valor social.

- La innovación social debe proveer a la comunidad de las herramientas para mantenerlo y evolucionarlo en el tiempo 
- $\quad$ La innovación, no debe ser patrimonio de quien la genera, sino que nace con vocación de ser difundida, reformulada y mejorada para que otros traten de adaptarla.

- $\quad$ La difusión y escala de la innovación social depende de la localidad en la que sea aplicada, pues la réplica implica realizar un nuevo análisis de la comunidad.

Lo anterior se complementa con el desempeño de la empresa en las dimensiones: económica, social y ambiental y su énfasis en la manera en que las organizaciones pueden y deben de atender a dichas dimensiones mientras se convierten en ágiles e innovadoras (FUNDACIÓN DE LA INNOVACIÓN BANKINTER, 2009) tal como se muestra en la Figura 3.

Figura 3 - Gestión de las expectativas económicas, medioambientales y sociales

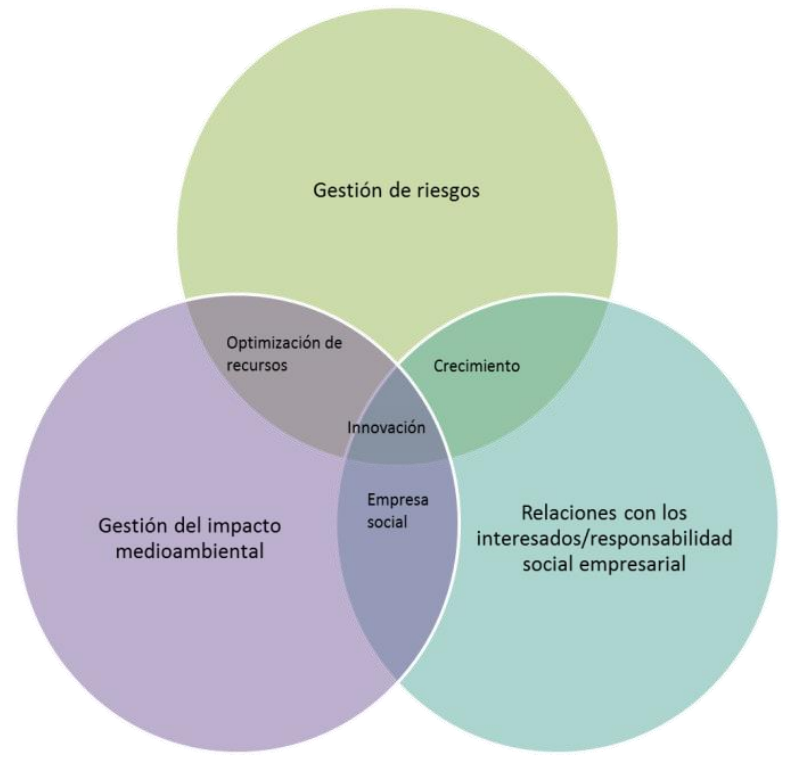

Fuente: Bankinter (2009).

Dicha gestión de las dimensiones implica que la organización para ser innovadora en el campo social requiere desarrollar alianzas y redes que permitan movilizar recursos, sobre todo conocimientos de diversos actores y agentes además de los propios principalmente con el sector público para ampliar el impacto de las innovaciones a cualquier escala, lo que a su vez implica la interacción entre dichos agentes. Por ello se debían especificar algunos criterios para seleccionar proyectos de innovación social (RODRÍGUEZ; ALVARADO, 2008) entre los que destacan: El beneficio social dirigido a un grupo significativo de personas, la originalidad, vigencia, 
consolidada, posibilidad de ser escalable y transformadora al tener impacto en otras variables de desarrollo social como costos, cobertura, ingreso, empleo, participación, rendimiento, cultura, calidad de vida entre otras.

De manera que la innovación es como la chispa creativa y original de una persona, grupo o entidad promotora, que se manifiesta en un hecho novedoso y transformador que desata progreso y permite utilizar mejor los recursos existentes; es un cambio en la práctica social que realiza una comunidad organizada, un proyecto del gobierno local o una organización no gubernamental con el fin de mejorar el impacto en la atención social o en la dinámica productiva del proyecto o práctica social (ASTORGA, 2004). Sin embargo, las entidades sociales también encuentran en su camino algunos obstáculos para la innovación, según menciona Rodríguez (2012):

- $\quad$ la falta de capacidad técnica;

- $\quad$ aversión al riesgo;

- $\quad$ estructuras de financiación;

- $\quad$ la desconfianza del equipo para innovar;

- $\quad$ la presencia de voluntarios y personal remunerado;

- $\quad$ falta de experiencia en innovación.

Ante dichos obstáculos las universidades juegan un papel fundamental al poder motivar desde el cumplimiento de su tercera misión al emprendimiento, la innovación y el compromiso social principalmente en el desarrollo del territorio o contexto en el que se asientan.

\section{Incubación de empresas sociales y el papel de la universidad}

El círculo de vida del emprendedor (DE PABLO et al., 2009) comienza cuando el emprendedor descubre una oportunidad y toma la determinación de que la oportunidad es válida para pasar a la elaboración de un plan de negocio, buscar financiación y finalmente la puesta en marcha de la empresa (antes de arriesgar dinero o recursos) por lo que el emprendedor puede pasar de la idea a la consolidación de la empresa pasando por los siguientes pasos:

1. La idea ha de cubrir una oportunidad de negocio, un hueco en el mercado o una necesidad insatisfecha o no cubierta. 
2. Realizar una búsqueda de información sobre la propia idea, analizando las posibilidades de llevarla a cabo y el impacto del entorno.

3. El plan de la empresa, documento en el que se plasman por escrito las actividades a llevar a cabo de una manera controlada por el emprendedor o empresario para la consecución de unos objetivos específicos y unos resultados concretos.

4. Búsqueda de fuentes de financiamiento

5. Consolidación de la empresa

Por lo anterior se puede observar que la misión de un programa de incubación de negocios es ayudar a los empresarios en la formación y el desarrollo de una nueva empresa para que esta pueda sobrevivir y ser exitosa. Para ello, un programa de incubación ofrece un marco de ayuda que facilita el proceso por el cual un empresario desarrollo un concepto de negocio y lo transforma en una empresa comercial viable. Según Lyons (2011) los programas de incubación deben alcanzar cuatro objetivos:

a) Incrementar la tasa de formación de nuevos negocios

b) Incrementar la tasa de supervivencia y éxito de las nuevas empresas.

c) Incrementar la tasa de desarrollo de nuevas empresas

d) Incrementar la eficiencia del proceso de disolución si una firma fracasa.

Cabe señalar que los programas de incubación más efectivos siguen estrategias específicas para agregar valor a la comunidad en la cual operan, lo cual es de suma importancia cuando se trata de emprendimientos sociales. El proceso de diseñar e implementar programas de incubación de negocios consiste en una serie de pasos que requiere de continuidad y un proceso de planificación adecuado que minimice el riesgo en las actividades que se desarrollarán.

Por ello una incubadora de empresas puede ser definida como un mecanismo de desarrollo empresarial que brinda una propuesta a la necesidad de mejoramiento productivo de la región, participando activamente en la creación de empresas, por lo que deben de constituir un ambiente que estimule la creatividad y la innovación que apunta a la cobertura de necesidades que los representantes del sector no pueden lograr de forma individual, tales como asesoramiento técnico, administrativo, contable, de costos, de mercado, de capacitación (GARCÍA, 2003). 
En México, de acuerdo con la Secretaría de Economía (SE, 2014) una incubadora de empresas es un centro de atención a emprendedores en donde te orientan y asesoran para que hagas realidad tu idea de negocio.

Otra definición de incubadora es un centro de apoyo que brinda asesorías especializadas para evaluar una idea de negocio y lograr que se convierta en una empresa formal (UNIVERSIDAD TECNOLÓGICA DE MÉXICO, 2014).

Una de las tareas de la incubadora es ayudar a preparar el plan de negocios y además, acompañan en el proceso de creación de las empresas, proporcionándoles consultoría en las diversas áreas que necesitas manejar al ser empresario: mercadotecnia, contabilidad, diseño gráfico e industrial. Asimismo, algunas incubadoras ofrecen espacios físicos para que emprender los negocios.

Cabe resaltar, que lo que te ofrece una incubadora no es financiamiento, sino la oportunidad de guiar a los futuros empresarios para enfrentar la vida de los negocios de manera más sólida y estructurada a través de la capacitación y consultoría especializadas.

\subsection{Tipos de incubadoras}

Existen tres tipos de incubadoras (SE, 2014):

1. Incubadora de Negocios Tradicionales. Apoya la creación de empresas en sectores tradicionales, cuyos requerimientos de infraestructura física, tecnológica y mecanismos de operación son básicos. Por ejemplo: restaurantes, papelerías, lavanderías, distribuidoras, comercializadoras, joyería, abarrotes y consultorías, entre otros.

2. Incubadora de Negocios de Tecnología Intermedia. Apoya la constitución de empresas cuyos requerimientos de infraestructura física y tecnológica y mecanismos de operación son semiespecializados e incorporan elementos de innovación. Por ejemplo: desarrollo de redes simples, aplicaciones web, tecnología simple para el sector alimentos, telecomunicaciones y software semiespecializados.

3. Incubadora de Negocios de Alta Tecnología. Apoya la constitución de empresas en sectores avanzados, tales como Tecnologías de la Información y 
Comunicación, microelectrónica, sistemas microelectromecánicos (MEM'S), biotecnología, alimentos y farmacéutico, entre otros.

Es muy importante señalar que las incubadoras operan en forma independiente y que cada una de ellas determina el costo por sus servicios. Sin embargo, gracias al apoyo de fondos público como el Fondo PYME, algunos de los servicios del proceso de incubación están subsidiados.

\subsection{Proceso de incubación}

El proceso de incubación de empresas, se divide en básicamente en tres fases (ver Figura 4):

- Pre-incubación: Canaliza los requerimientos que se necesitan para poder incubar un proyecto, cumplir con los requisitos necesarios; así como tener las herramientas necesarias para iniciar su proceso de incubación.

- Incubación: El proceso de incubación inicia con la prueba y la validación de su proyecto, seguido del objeto del negocio, creación, pilotaje y lanzamiento o alumbramiento del mismo.

- Pos-incubación: Consiste en no perder el contacto con el incubado para saber cómo sigue el proyecto, cuáles han sido sus logros dentro del mercado.

Figura 4 - Proceso de incubación de empresas

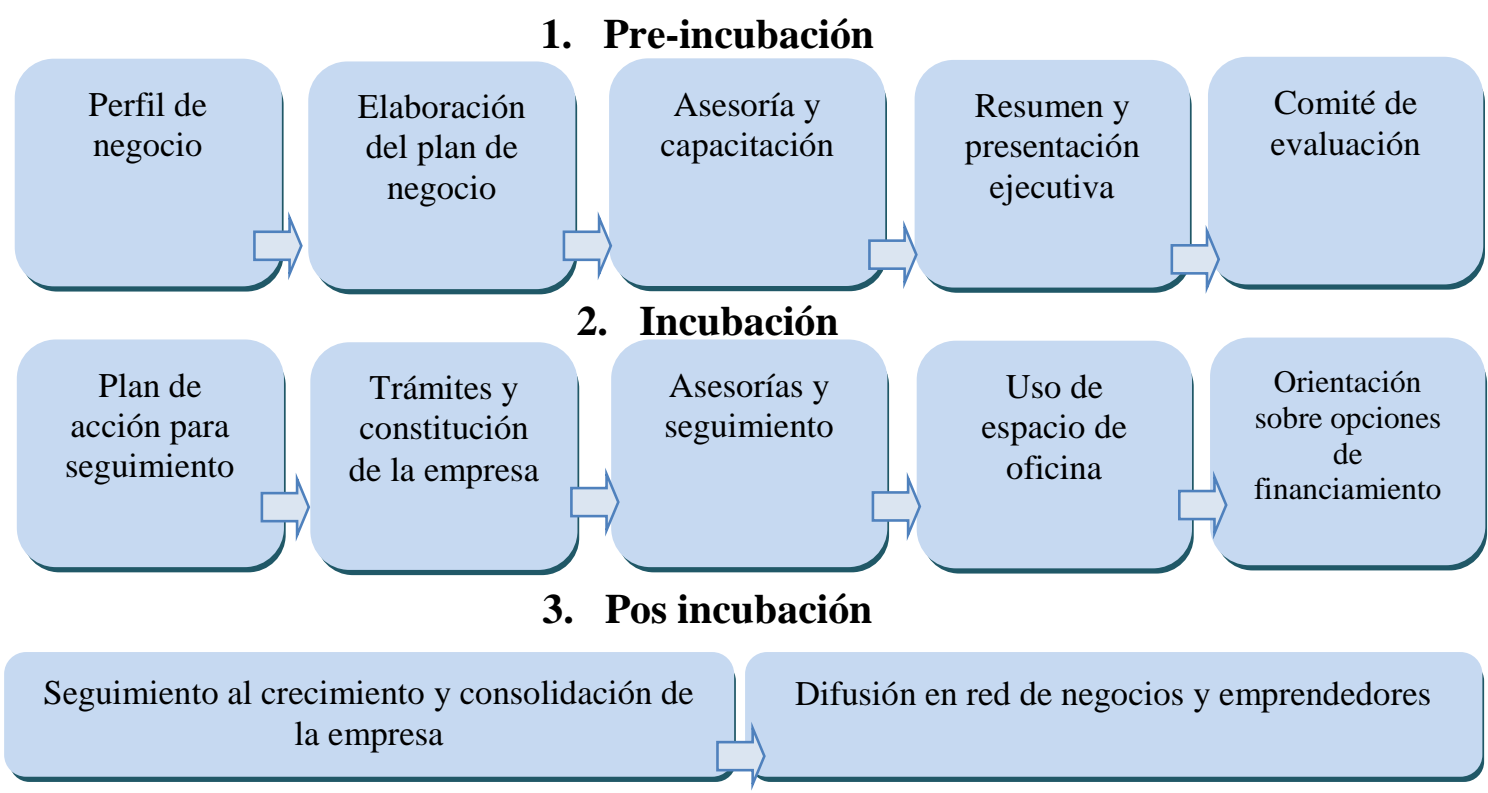

Fuente: elaboración propia con base en Universidad Tecnológica Emiliano Zapata del Estado de Morelos, UTEZ (2014). 
Relacionando el proceso de incubación al emprendimiento social, las incubadoras sociales acompañan la creación de empresas a través de una metodología específica que posibilite la organización e inserción de trabajadores y producir una mejora significativa en ellos.

Desde la universidad las incubadoras permiten transferir conocimientos tecnológicos de gestión de producto o procesos para sectores que por distintos motivos, padecen dificultades de acceso a empleo. La inserción de la universidad en las temáticas de la pobreza y el desempleo a través de las incubadoras de empresas sociales, aparece como la posibilidad de ofrecer una respuesta práctica a las necesidades de la población que requiere del acompañamiento de estrategias para enfrentar el empleo y dar solución a otras problemáticas sociales.

Por ello las universidades desde su tercera misión aluden a la transferencia de conocimiento que se concibe como el movimiento y difusión de una tecnología o producto desde su invención original a un contexto económico y social diferente por lo que la transferencia puede ser no comercial, comercial y de creación de nuevas empresas (ARIAS; ARISTIZÁBAL, 2011). El primero se refiere básicamente a la difusión del conocimiento sin ningún tipo de interés comercial, el segundo cuando la transferencia se celebra bajo un acuerdo comercial entre la universidad y su contraparte a través de capacitaciones o investigación conjunta y comercialización de licencias o bien mediante la creación de spin-offs. Sin embargo la incubación juega un papel importante al apoyar a dicha transferencia.

Por otra parte las universidades pueden desarrollar diversas acciones de comunicación y difusión sobre el concepto de innovación social para informar a la población y favorecer su participación, general diálogos interdisciplinarios, impulsar el movimiento estudiantil para la resolución de retos sociales del territorio y propuestas de cambio.

Principalmente porque el emprendedor social debe ser consciente y sensible hacia grupos sociales marginados, vulnerables. Por ello diversos programas e incubadoras en universidades pueden y deben contribuir a desarrollar este enfoque direccionando el proceso de enseñanza/aprendizaje en el fortalecimiento del individuo 
como ente social enfocándose en el ser para desarrollar habilidades y acercarlo a la sociedad para que conozca los problemas y sus necesidades.

\subsection{Principales incubadoras en el estado de Hidalgo}

De acuerdo con los datos del Instituto Nacional de Incubadoras de Empresas (2014), en México existen 216 incubadoras tradicionales, diez de ellas se encuentran en el estado de Hidalgo, de las cuales el $84.61 \%$ están ubicadas en instituciones educativas de nivel superior, lo que facilita el acercamiento de los estudiantes.

De acuerdo con información de la Secretaria de Economía (2014), el estado de Hidalgo, es una de las seis entidades federativas que han registrado un número mayor de incubadoras de empresas ante el Instituto Nacional del Emprendedor.

Cabe hacer mención, que a partir del año 2011, se crea el Centro Emprendedor de Universidades Politécnicas y Tecnológicas en el estado de Hidalgo, que tienen como modelo de incubación de tecnología intermedia reconocida y que apoyan, principalmente, proyectos de investigación de alumnos y/o maestros que ayuden a cristalicen sus ideas en empresas reales y exitosas.

Por otra parte, el Instituto Politécnico Nacional, a partir de enero del 2014, puso en servicio su Centro de Incubación de Empresas en el Estado de Hidalgo, con el objetivo de impulsar proyectos productivos que se realizan dentro de sus aulas y que cuenten con características de impacto social basados en la tecnología y lo más importante, que dichos proyectos sean realizados por recién egresados o que se encuentren a punto de egresar. Aunque es importante destacar que dichos proyectos serán guiados desde el Centro de Investigación y de Estudios Avanzados del Instituto Politécnico Nacional (CINVESTAV), en la ciudad de México.

\section{Resultados}

Para la presente investigación, se llevó a cabo una revisión sobre la literatura existente del tema de innovación social e incubadoras, esto, con el fin de comprender mejor estos conceptos, así como su relación con las universidades y su importancia para la competitividad del Estado de Hidalgo.

Asimismo, a través de la búsqueda en distintas bases de datos, se identificaron las principales acciones desarrolladas por las incubadoras sociales más importantes del 
estado de Hidalgo, durante los últimos tres años (2011-2014). Lo anterior con el fin de conocer los resultados más significativos y de mayor impacto para el Estado.

De las principales incubadoras sociales que se encuentran en el estado de Hidalgo, con base en la investigación documental se encuentran los siguientes resultados:

El $100 \%$ de las incubadoras sociales proporciona los conocimientos necesarios para el desarrollo de empresas mediante la asesoría de acompañamiento y adiestramiento de negocios en diferentes áreas, entre las cuales se encuentran principalmente en el área de Tecnología de Información y Comunicación, Acevedo (2013) y en menor escala empresas de base social, estableciendo un modelo de negocios, que permitan a las empresas creadas ser competitivas en el mercado.

En cuanto a los casos de éxito de las principales incubadoras sociales establecidas en instituciones de educación superior, de acuerdo a la investigación documental, la Incubadora de la Universidad Autónoma del Estado de Hidalgo, durante este año, ha apoyado a más de 220 emprendedores a formar su empresa, de las cuales 20 son dedicadas a las actividades sociales, solo en la capital del estado, esto se debe al trabajo realizado conjuntamente con la Fundación Hidalguense y el Patronato Universitario con otras instituciones y asociaciones civiles a través de proyectos tales como la reinserción social de menores infractores, Fomento al Emprendimiento de Negocios Sociales por Jóvenes Universitarios Indígenas, para estudiantes de zonas rurales, Programa de Innovación para el Desarrollo Equitativo donde se otorga apoyo a mujeres artesanas así como recientemente el Programa de Jóvenes Defensores de los Derechos de las personas con discapacidad UAEH (2014).

De la misma forma, la Incubadora del Instituto Tecnológico de Estudios Superiores de Monterrey, Campus Hidalgo, la cual su modelo de incubación es de tecnología intermedia y base social han permitido que sus alumnos, durante el año 2013, sean finalistas en VI Premio Santander a la Innovación Empresarial con el proyecto VPark, Adicionalmente, han creado seis empresas de base social, reconocidas a nivel estatal, que se dedican desde la elaboración de comida delicatesen hasta empresas especialistas en el desarrollo de software (ITESM, 2014). 
Finalmente, otro caso de éxito de las empresas sociales, en el estado de Hidalgo, reconocida por la Revista Forbes (2013) es el Parque Ecoturístico y Acuático Ecoalberto, situado en el municipio de Ixmiquilpan, Hgo., donde un grupo de indígenas Hñahñu (otomí), se organizaron para crear un parque comunitario, que les permita generar trabajo a su comunidad y, evitar de esta forma la emigración a los Estados Unidos.

Esta organización comunitaria ha desarrollado un verdadero complejo turístico, que explota de una manera ecológica todos sus atractivos naturales. Los visitantes pueden hospedarse en cabañas construidas con materiales de la región, como piedra, madera y penca de maguey.

Además, cuentan con un programa de operación ecológica, que incluye una planta de tratamiento de aguas residuales, manejo de desechos orgánicos e inorgánicos y acciones de concientización para el cuidado y respecto de los recursos naturales de la región.

\section{Conclusiones}

La innovación social busca obtener - en la medida de lo posible - resultados positivos para la sociedad, no obstante, pueden existir sociedades con un comportamiento conformista y con una fuerte resistencia al cambio.

Cabe destacar que la innovación social aborda una variedad de herramientas que crea una oportunidad para un ejercicio a gran escala, ya que una serie de innovaciones sociales puede iniciar a nivel local, donde tienen que encontrar apoyo y capacidades; y deben ser alentados a nivel regional y nacional, donde las opiniones convergen en el papel decisivo de la difusión de buenas prácticas y promover el cambio. El éxito de la innovación social se basa en la calidad de las asociaciones horizontales y verticales entre diversos actores.

Es pertinente recordar en este punto, que la variedad de contextos influye en la adopción de nuevos procesos y su impacto. En ese tenor, se puede argumentar que las universidades son pieza clave para una mejor absorción de innovaciones sociales, debido a su papel en la generación de conocimiento y de su influencia para renovar su modelo social desde la academia hacia la sociedad. 
Sin embargo, en cuanto a la educación y a las habilidades para el desarrollo, los retos de la sociedad exigen una reforma profunda de los sistemas educativos. Esto implica una mayor interdisciplinariedad, una interacción más fuerte entre la investigación básica y aplicada, una mayor rendición de cuentas y una mayor comprensión de los verdaderos impactos sociales de los desarrollos tecnológicos, tanto en términos de oportunidades como en términos de amenazas. Cuestiones tales como la responsabilidad social y los impactos críticos del desarrollo tecnológico, serían parte de ello.

Por otra parte, por la importancia que la educación desempeña para proporcionar las habilidades que necesitan los emprendedores para hacer frente a los retos sociales, cuestiones como la falta de financiación y la falta de rendición de cuentas también pueden frenar esas iniciativas.

Los resultados de esta investigación permitieron reconocer la labor que realizan las incubadoras sociales de las Universidades, en atención a las problemáticas sociales de Hidalgo. Entre los resultados más sobresalientes, se tiene: la inclusión de personas con discapacidad y el emprendimiento en regiones indígenas en la que se promueve el diseño e implementación de mejores maneras de cubrir necesidades de colectivos empobrecidos y marginados lo que permite favorecer el crecimiento personal y colectivo de las regiones y la equidad.

Finalmente, es necesario mencionar que la adopción generalizada de innovaciones sociales necesita ser apoyada y acelerada, y que gran parte de este trabajo debe hacerse a nivel nacional, regional y local, ya que debe hacerse un mayor uso de las políticas y los instrumentos de financiación para crear las condiciones en las que la innovación social se convierte en un activo medible para el Estado de Hidalgo.

\section{REFERENCIAS}

ACEVEDO, G. Activará IPN Centro de Incubación de Empresas. 2013. Disponible en: $<$ http://www.milenio.com/region/Activara-IPN-Centro-IncubacionEmpresas_0_184781557.html>.Acceso en: 14 ago. 2014.

ARIAS, P.; ARISTIZÁBAL, B. Transferencia de conocimiento orientada a la innovación social en la relación ciencia-tecnología y sociedad. Pensamiento y Gestión, n. 31. 
ASTORGA, E. Innovación social. Concepto, criterios y variables. Experiencias en innovación social en América Latina y el Caribe. Chile, 2004.

BOWNS, C.; STEVENSON, A. Toward small town revitalization in the Middle Susquehanna River Valley: Re-assessing historic and regional resources for collaborative development. Material Culture, v. 42, n. 2, p. 1-27, 2010.

BUENO, C. E. La tercera misión de la universidad: El reto de la Transferencia del conocimiento.

2007.

Disponible

en: <http://www.madrimasd.org/revista/revista41/tribuna/tribuna2.asp>. Acceso en: 20 mai. 2014.

CENTRO DE INNOVACIÓN Y LIDERAZGO EMPRENDEDOR. (2014). Incubadora de Empresas. Instituto Tecnológico de Estudios Superiores de Monterrey. Disponible en: <http://prod03.hgo.itesm.mx/cile/web/index.php/areas-estrategicas/incubadora-de-empresas>. Acceso en: 24 ago. 2014

CENTRO INCUBADOR DE EMPRESAS. (2014). Logros en Vinculación Social. Universidad Autónoma del Estado de Hidalgo. Disponible en: <http://www.uaeh.edu.mx/adminyserv/gesuniv/div_vin/dir_socprod/logros.html>. Acceso en: 2 sep. 2014.

CONSEJO DE COMUniCACiÓn. (2014). Pepe y Toño. Consigue el apoyo que necesitas. Disponible en: <http://www.pepeytono.com.mx/novedades/2012/11/consigue_el_apoyo_que_necesitas〉. Acceso en: 12 jun. 2014.

CONSEJO ESTATAL DE POBLACIÓN. (2014). Índice y grado de marginación de Hidalgo. Disponible en: $<$ http://poblacion.hidalgo.gob.mx/index.php?option=com_content\&task=view\&id=18>. Acceso en: 12 jun. 2014.

DE PABLO, V.; URIBE T. Emprendimiento de la economía social y desarrollo local: la promoción de incubadoras de empresas de economía social en Andalucía. CIRIEC, revista de economía pública, social y cooperativa, n. 64, 2009.

EMPRENDEDOR, I. N. Instituto Nacional de Emprendimiento. Disponible en: <https://www.inadem.gob.mx/incubadoras_y_aceleradoras.html>. Acceso en: 3 sep. 2014.

ENCISO S.; GÓMEZ, U. La iniciativa comunitaria a favor del emprendimiento social y su vinculación con la economía social: una aproximación a su delimitación conceptual. CIRIEC, revista de economía pública, social y cooperativa, n. 75, p. 55-80.

R. Bras. Planej. Desenv., Curitiba, v. 4, n. 1, p. 128-148, jan./jun. 2015. 
GALINDO, M. J.; SANZ, A. P. La universidad ante el reto de la transferencia del conocimiento 2.0.: Análisis de las herramientas digitales a disposición del gestor de transferencia. Investigaciones Europeas de Dirección y Economía de la empresa, v. 17, n. 3, p. 111-126, 2011.

GARCÍA F. M. Incubación. Entrepreneur, v. 20, n. 3, p. 70, 2012.

GARCÍA, A. La incubadora de empresas, una alternative para fomenter el desarrollo empresarial en el estado de Oaxaca. Universidad Tecnológica de la Mixteca, 2003. Disponible en: <http://jupiter.utm.mx/ tesis_dig/8306.pdf〉. Acceso en: 15 jun. 2014.

GOBIERNO DE MÉXICO. Hidalgo y su actividad económica. Secretaría de Economía, 2014. Disponible en: <http://www.economia.gob.mx/delegaciones-de-la-se/estatales/hidalgo\#>. Acceso en: 3 sep. 2014.

GUZMÁN, V.; TRUJILLO, D. Emprendimiento social - revisión de literatura. Estudios Gerenciales, v. 24, n. 109, p. 105-125, 2008.

HAYBRON, D. M. Philosophy and the science of subjective well-being. In: EID, M.; LARSEN, R. J. (Eds.) The science of subjective well-being. New York, NY: Guilford Press, 2008. p. 17-43.

JUDD, D. M. A history of American nursing: Trends and eras. Sudbury, MA: Jones and Bartlett, 2007.

KANTIS, H. Indice de condiciones sistémicas para el emprendimiento dinámico: una herramienta para la acción en América Latina. 1.ed. Edición. Rafaela: Asociación Civil Red Pymes Mercosur, 2014.

LOYOLA, J. E. L. El "reality show" de los emigrantes mexicanos. Revista Forbes, 2013. Disponible en: <http://www.forbes.com.mx/el-reality-show-de-los-migrantes-mexicanos/>. Acceso en: 25 ago. 2014.

LYONS, S. T.; LICHTENSTEIN G. A. Incubando nuevas empresas. Disponible en: <http://ucapanama.org/wp-content/uploads/2011/12/Incubando-Nuevas-Empresas.pdf>. Acceso en: 15 jun. 2014.

MARTÍNEZ, R.; RODRÍGUEZ, D. Emprendedurismo social en México: hacia un modelo de innovación para la inserción social y laboral en el ámbito rural. Procuraduría Agraria, p. 103$120,2013$. 
MERINO, A. et al. Guía del emprendedor social. Inspiraciones para la creación de empresas al servicio de la sociedad. Universidad Pontificia, España, 2013.

MORIANO, J. A. El perfil psicosocial del emprendedor. Consejo Económico y Social, Madrid, 2005.

ORGANISATION FOR ECONOMIC CO-OPERATION DEVELOPMENTE (OECD). SMEs, entrepreneurship and innovation. 2010.2 Disponible en: <http://www.oecd.org/cfe/smesentrepreneurshipandinnovation.htm>. Acceso en: 15 jun. 2014.

RODRÍGUEZ, B. E.; CARRERAS I. Innovar para el cambio social. De la idea a la acción. ESADE. Instituto de Innovación Social. 2012. Disponible en: $<$ http://itemsweb.esade.es/wi/research/iis/publicacions/2012-InnovarParaCambioSocialweb.pdf>. Acceso en: 24 feb. 2013.

RODRÍGUEZ, H.; ALVARADO, U. Claves de la innovación social en América Latina y el Caribe. CEPAL/Naciones Unidas, 2008.

SALINAS, F.; OSORIO, L. Emprendimiento y economía social, oportunidades y efectos en una sociedad en transformación. CIRIEC, Revista de Economía Pública, Social y Cooperativa, v. 75, p. 129-151, 2012.

SHANE, S.; VENKATARAMAN S. The promise of entrepreneurship as a field of research. Academy of Management. The Academy of Management Review, v. 25, n. 1, p. 217, 2000.

SPIVA, M. Development of coping strategies used by parents of children with autism. (Unpublished master's thesis). California State University Stanislaus, Turlock, CA, 2010.

VILALTA, M. J. La tercera misión universitaria. Innovación y transferencia de conocimientos en las universidades españolas. Cuadernos de trabajo, n. 3, 2000.

WESTLEY, F.; ANTANDZE, N. Making a difference: Strategies for Scaling social innovation for greater impact. The Innovation Journal: The Public Sector Innovation Journal, v. 15, n. 2,2010

Recebimento dos originais: 07/05/2015

Aceitação para publicação: 27/08/2015 